\title{
Surgical Management of Sequelae Caused by Septic Hip Arthritis in Children
}

\author{
Andrei Maxim*, Dragoș Apostu and Dan Cosma \\ University of Medicine and Pharmacy Cluj-Napoca, Romania \\ *Corresponding author: Andrei Maxim, Department of Pediatric Orthopedics, Romania
}

\section{ARTICLE INFO \\ Received: March 19, 2019 \\ Published: 蔧 March 26, 2019 \\ Citation: Andrei Maxim, Dragoș Apos- tu, Dan Cosma. Surgical Management of Sequelae Caused by Septic Hip Arthri- tis in Children. Biomed J Sci \& Tech Res 16(3)-2019. BJSTR. MS.ID.002847.}

Keywords: Pediatric septic hip arthritis; Sequelae hip arthritis; Reconstruction of the hip in children

\begin{abstract}
Objective: Septic arthritis is one of the diseases with a high destructive effect on the articular and bone level. A delay in diagnosis or a misdiagnosed septic arthritis of the pediatric hip it can be a complicated challenge for many physicians. The aim of this study was to evaluate surgical options for the management of sequelae caused by septic arthritis in children.
\end{abstract}

Material and Methods: We used the electronic databases and selected articles that had as main topic the septic arthritis of the hip in children and their methods of diagnosis and treatment.

Results: We can use IL-6 and CRP (C- Reactive Protein) for early diagnosis. The most important thing is early diagnosis and early treatment to prevent premature closure of the triradiate cartilage, acetabular dysplasia, subluxation, dislocation and ischemic necrosis of the femoral head.

Conclusion: Good early diagnosis and appropriate treatment are required for a good result. Management of sequelae after hip septic osteoarthritis is a difficult and long-lasting one and that does not always manage to correct everything that has been destroyed.

\section{Introduction}

Septic arthritis represents a bacterial infection of the synovium followed by impairment of all components of the joint, causing a strong inflammatory reaction, destruction of the articular cartilage and later destruction of the complete joint. This can be a primary infection or occur because of osteomyelitis with spread into adjacent joint. The main cause is haematogenous dissemination of the bacteria and only in few cases by direct inoculation [1]. There are several known risk factors for septic arthritis: male gender, young age, umbilical artery catheterisation, respiratory distress syndrome, musculoskeletal intervention, host phagocystic defects haemoglobinopathies, interventions on joints, instrumentation of the urinary and intestinal tract [1-3]. Majority of children presents in the emergency room and they acuse limping, fever, poor general condition, impaired range of motion and pain, refuse to bear weight but in neonates they can present pseudo paralysis of the affected limb or paradoxical irritability $[1,4]$. The diagnosis it's important to be made quickly and treatment should start immediately.

The factors that negatively influence the evolution of septic arthritis are delay in diagnosis and treatment, age at onset of infection, premature birth, and association with osteomyelitis [4]. Delay in diagnosis and treatment will make sequelae of septic arthritis inevitable. Septic arthritis is a diagnostic and therapeutic emergency. Patients between 3 -18 years usually develop recurrent infections with femoral head destruction and secondary hip dislocation. The evolution and prognosis of septic arthritis depends on two main factors: early diagnosis and appropriate treatment [5]. The most frequent joint affected are hip joint and knee joint. The microorganisms most commonly involved in septic osteoarthritis are: Staphylococcus aureus, Streptococcus pneumoniae and Hemophilus influenzae [6]. Septic arthritis causes irreversible 
cartilage or bone destruction with permanent joint dysfunction. The most common complications are premature closure of the triradiate cartilage, premature closure of the proximal femoral physis, limb length inequality, subluxation, dislocation and complete destruction of the femoral head and neck [7].

\section{Objective}

The purpose of this study is to evaluate surgical techniques for management of sequelae of the hip joint after septic osteoarthritis depending on the degree of severity of the disease.

\section{Material and Methods}

\section{Search Strategy}

We used electronic database MEDLINE (Medical Literature Analysis and Retrieval System Online) trought PubMed interface and Google Books search engine. The keywords and Medical Subject Heading (MeSH) terms used were pediatric septic hip arthritis, sequelae hip arthritis, reconstruction of the hip in children.

\section{Inclusion and Exclusion Criteria}

Inclusion criteria were full text articles, septic joint hip reconstruction in children, strategies for treatment and diagnosis of septic arthritis of the hip in children and exclusion criteria were studies about adult hip pathology.

\section{Results}

It is known that early diagnosis of hip arthritis is a challenge for physicians. The differential diagnosis of irritable hip includes infection, trauma, inflammatory arthritis, vascular or neoplastic pathology. Most of them can be excluded with a complete and good clinical examination, and with aid of imaging. Normal value of CRP (C-Reactive Protein) has a high predictive value for excluding septic arthritis [1,2]. Fatima F et al. [8] tried to associate some biomarkers with bone erosion in septic arthritis in study on mice. The biomarkers studied was cross-linked carboxyterminal telopeptide of type I collagen (ICTP), a bone turnover marker used in early evaluation of skeletal metastases in cancer patients, and IL-6. The result of study was that ICTP is not a biomarker for bone destruction, but it showed a good correlation between weight loss and serum level of IL-6, that suggests IL- 6 may be used as a biomarker for bone erosion in septic arthritis. Also, they studied the radiological features of bone destruction in mice with septic arthritis using $\mu \mathrm{CT}$ and they tried to find a correlation between data obtain by $\mu \mathrm{CT}$ and histological evaluation of bone tissue. They observed an excellent correlation between $\mu \mathrm{CT}$ data and histological evaluation of septic arthritis and demonstrate the accuracy and sensitivity of $\mu$ CT [8].

Choi IH et al. [9] in a study published in The Journal of Bone and Joint Surgery in 1990, evaluated the sequelae and delay in treatment in children who had had septic arthritis when they had one year old or less. Study included thirty-one children with thirtyfour hips affected by septic arthritis. To evaluate the deformities and sequelae after septic arthritis of the hip joint they use Hunka classification [10]. The average duration of follow-up was twelve years and they evaluate: pain, range of motion, limb-length discrepancy and radiographic evaluation of hip joint. In type I they done aspiration of the hip joint and intravenous administration of antibiotics, and the hips was protected by containment in abduction cast or brace for an average of 1.2 years. In type II the deformities are abnormality of the size and shape of the femoral head, shortened length of the femoral neck, modify of the femoral neck-shaft angle and a major limb-length discrepancy. In this case is necessary closed follow-up during growth, because in the presence of radiographic signs of avascular necrosis, the femoral head should be protected by abduction in a cast or orthosis.

If the subluxation of the hip is present, acetabuloplsty or proximal femoral osteotomy may be necessary to provide adequate coverage of the femoral head and re-establish congruency of the femoral head and acetabulum. Type III presents coxa vara or coxa valga associate with extreme anteversion or retroversion, or pseudarthrosis of the femoral neck with complete epiphyseal slipping. In this case was made subtrochanteric osteotomy, in case of pseudarthrosis bone-grafting, Pemberton acetabuloplasty in case of acetabular deficiency and epiphysiodesis to the distal part of contralateral femur to prevent limb-length discrepancy. In type IV there is a complete destruction of the femoral head and neck, severe limb-length discrepancy, acetabular dysplasia. There were used three methods to manage this hip: trochanteric arthroplasty with adjunctive and secondary procedures, arthrodesis and observation without surgical treatment. In type IV the only success method was trochanteric arthroplasty, also they suggest that trochanteric arthroplasty should be combined with vasur osteotomy or pelvic osteotomy to obtain a better coverage of the proximal femur10. The optimal age for performing these procedures was not established. Are studies that recommend that these procedures be performed in children under the age of 2 to allow them to develop their gait and to allow the physician to follow the evolution of the length of the limbs $[10,11]$.

Greater trochanteric preserving hip arthroplasty was evaluated in a study, published in J Child Orthoepy in 2010, for treatment of hips with Choi type IVB residual deformities [12]. Inclusion criteria was: patients younger than 3 months of age when the septic arthritis developed, complete destruction of the femoral head and neck and more than ten years follow-up. All patients were treated with the same treatment method. The follow-up evaluation included: pain, range of motion, gait analysis, limblength discrepancy, final coverage and stability of the hip joint. All patients had a pain-free hip at the final evaluation and achieved a near-normal level of daily activities. The result was classified as satisfactory after criteria Hunka et al. [10,12]. The surgical success in this intervention is the stabilization of the hip joint, small differences in length between the limbs, all of which lead to better conditions of total replacement of the hip [13]. Transposition of the apophysis of the greater trochanter for the reconstruction of 
the femoral head after septic arthritis was also studied and results followed-up more than 15 years. In this study was included four children between 1-6 years old with a complete necrosis of rhea femoral head after septic arthritis. They were operated on with transposition of the greater trochanter. Radiographic postoperative evaluation showed development of a new femoral head in all hips. Limb-length discrepancy was corrected with distal femoral epiphysiodesis and lengthening osteotomy of the femur. The result of this treatment was hips without pain and with a mobility that allowed good walking function [14].

It is known that hip joint presents a severe instability like a consequence after septic arthritis in children and its management remain a challenge. The pelvic support osteotomy was described to resolve hip instability. Ilizarov modified technique and added a second, more distal varus femoral osteotomy for lengthening to restore the overall alignment of the lower limb. A retrospective study evaluated midterm results of the Ilizarov hip reconstruction. The inclusion criteria were minimum postoperative follow-up 5 years, unilateral hip instability after septic arthritis in childhood. The presenting symptom were a limp, leg-length discrepancy and daily activity including difficulty with marital relationship. The functional outcome was graduated using the Harris hip score. The result of this treatment had had a good result in $81,25 \%$. Improvement in the functional outcome was statistically significant and limb-length discrepancy was diminished [15].

\section{Conclusion}

Septic arthritis still remain a challenge to be diagnose and to treat. A delay of diagnosis of more than 4 days will lead to the destruction of the hip joint from the simplest form of treatment to interventions at the proximal femur and interventions on the pelvis with reconstructions, arthroplasty to achieve a stable, functional and painless hip. As studies have shown us that CRP (C-Reactive Protein) can be used as the presence of septic arthritis and IL- 6 can be used as a marker for assessing bone erosion in septic arthritis. CT scan can be used to assess bone destruction and evaluating the remaining bone stock. In the early stages of the disease intravenous drug treatment and immobilization of the joint for femoral head protection has a beneficial effect, but the radiological follow-up of the hip joint is mandatory. In advanced stages in children (Hunka type IV-V or Choi V) transposition of the apophysis of the greater trochanter for the reconstruction of the femoral head remain a good option with good results for a long period of time. For late sequelae of septic arthritis of the hip Ilizarov hip reconstruction bring good advantages like hip stabilizing, decrease the limb-length discrepancy and obtain a functional hip joint.

\section{References}

1. Rutz E, Spoerri M (2013) Septic Arthritis of the Pediatric Hip-A Review of Current Diagnostic Approaches and Therapeutic Concepts. Acta Orthop Belg 79(2): 123-134.

2. Kang SN, Sanghera T, Mangwani J, Paterson JM, Ramachandran M (2009) The Management of Septic Arthritis in Children: Systematic Review of the English Language Literature. J Bone Joint Surg Br. 91(9): 1127-1133.

3. Shah SS (2005) Abnormal Gait in a Child with Fever: Diagnosing Septic Arthritis of the Hip. Pediatr Emerg Care 21(5): 336-341.

4. Gang Xu, Spoerri M, Rutz E (2016) Surgical Treatment Options for Septic Arthritis of the Hip in Children. Afr J Paediatr Surg 13(1): 1-5.

5. Anil A, Aditya NA (2013) Pediatric Osteoarticular Infection. Edition Jaypee Brothers Medical Publishers, p. 75-78.

6. Young TP, Maas L, Thorp AW, Brown L (2011) Etiology of Septic Arthritis in children: An Update for the New Millennium. Am J Emerg Med 29(8): 899-902.

7. Cheung A, Lam A, Ho E (2010) Sonography for the Investigation of a Child with a Limp. Australas J Ultrasound Med 13(3): 23-30.

8. Fatima F, Fei Y, Ali A, Mohammad M, Malin C Erlandsson, et al. (2017) Radiological Features of Experimental Staphylococcal Septic Arthritis by Micro Computed Tomography Scan. PLoS One 12: e0171222.

9. Choi IH, Pizzutillo PD, Bowen JR, Dragann R, Malhis T (1990) Sequelae and Reconstruction After Septic Arthritis of the Hip in Infants. J Bone Joint Surg Am 72(8): 1150-1165

10. Hunka L, Said SE, MacKenzie DA, Rogala EJ, Cruess RL (1982) Classification and Surgical Management of the Severe Sequelae of Septic Hips in Children. Clin Orthop Relat Res 171: 30-36.

11. Harmon PH (1942) Surgical Treatment of the Residual Deformity from Suppurative Arthritis of the Hip Occurring in Young Children. J Bone and Joint Surg 24(3): 576-575.

12. Abrishami S, Karami M, Karimi A, Soufali AP, Aslani HR, et al. (2010) Greater Trochanteric Preserving Hip Arthroplasty in the Treatment of Infantile Septic Arthritis: Long-Term Results. J Child Orthop 4(2): 137141.

13. Freeland AE, Sullivan DJ, Westin GW (1980) Greater Trochanteric Hip Arthroplasty in Children with Loss of the Femoral Head. J Bone Joint Surg Am 62(8): 1351-1361.

14. Pål Benum (2011) Transposition of the Apophysis of the Greater Trochanter for Reconstruction of the Femoral Head After Septic Hip Arthritis in Children. Acta Orthop 82: 64-68.

15. Mahmoud A El-Rosasy, Mostafa A Ayoub (2014) Midterm Results of Ilizarov Hip Reconstruction for Late Sequelae of Childhood Septic Arthritis. Strategies Trauma Limb Reconstr 9(3): 149-155.

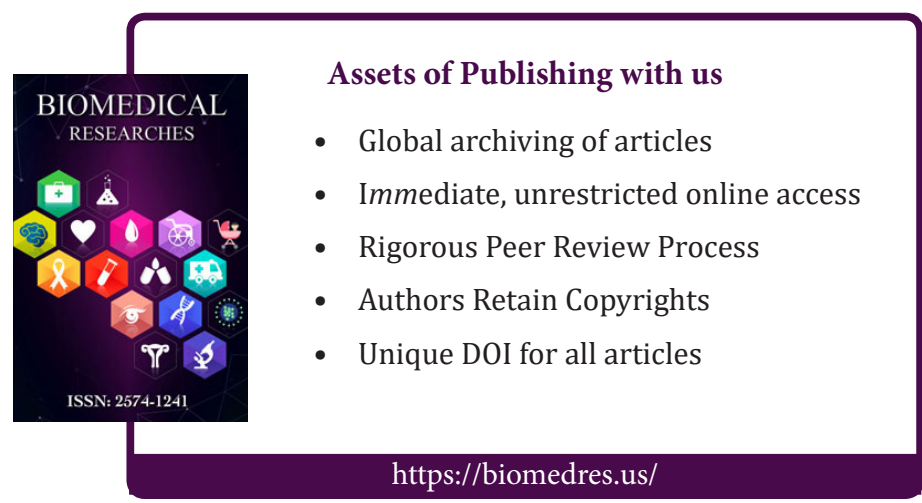

